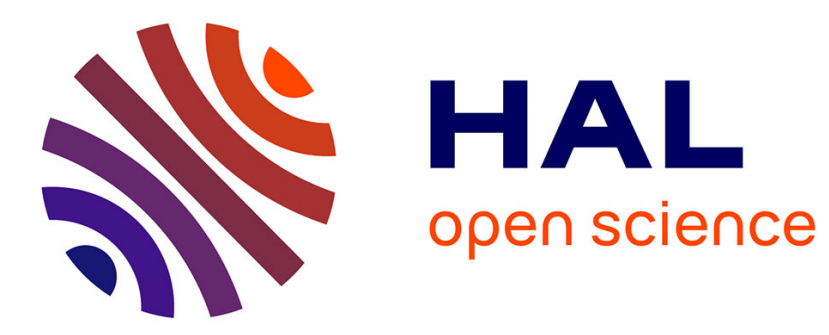

\title{
Multicomponent superconductivity: The complex interplay of spin charge and lattice
}

\author{
Annette Bussmann-Holder
}

\section{To cite this version:}

Annette Bussmann-Holder. Multicomponent superconductivity: The complex interplay of spin charge and lattice. Philosophical Magazine, 2006, 86 (13-14), pp.1793-1806. 10.1080/14786430500300108. hal-00513600

\section{HAL Id: hal-00513600 \\ https://hal.science/hal-00513600}

Submitted on 1 Sep 2010

HAL is a multi-disciplinary open access archive for the deposit and dissemination of scientific research documents, whether they are published or not. The documents may come from teaching and research institutions in France or abroad, or from public or private research centers.
L'archive ouverte pluridisciplinaire HAL, est destinée au dépôt et à la diffusion de documents scientifiques de niveau recherche, publiés ou non, émanant des établissements d'enseignement et de recherche français ou étrangers, des laboratoires publics ou privés. 


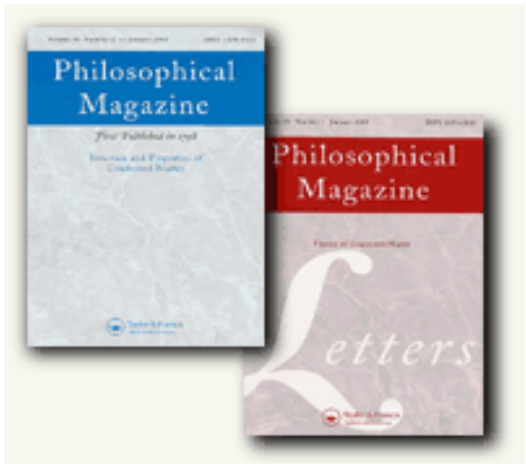

\section{Multicomponent superconductivity: The complex interplay of spin charge and lattice}

\begin{tabular}{|r|l|}
\hline Journal: & Philosophical Magazine \& Philosophical Magazine Letters \\
\hline Manuscript ID: & TPHM-05-Mar-0063 \\
\hline Journal Selection: & Philosophical Magazine \\
\hline Date Submitted by the \\
Author: & 02-Mar-2005 \\
\hline Complete List of Authors: & $\begin{array}{l}\text { Bussmann-Holder, Annette; Max-Planck-Institute for Solid State } \\
\text { Research }\end{array}$ \\
\hline Keywords: & high-temperature superconductivity, superconductivity \\
\hline Keywords (user supplied): & \\
\hline
\end{tabular}

\section{$\checkmark$ ScholaroNE" \\ Manuscript Central}




\title{
Multicomponent superconductivity: The complex interplay of spin charge and lattice
}

\author{
Annette Bussmann-Holder, Max-Planck-Institute for Solid State Research, Heisenbergstr.1, \\ D-70569 Stuttgart, Germany
}

High temperature superconducting copper oxides (HTSC) have been shown to be intrinsically heterogeneous. Consequently multiple ground states coexist, which are either in competition or of support to superconductivity. Here it is shown that spin, charge and lattice are intrinsically coupled to form a composite of locally charge carrying distorted areas in an antiferromagnetic matrix on the regular lattice. The dynamical interplay between both induces level shifts together with band narrowing of the spin and charge related bands, by means of which interband interactions are possible which lead to a substantial increase in the superconducting transition temperature $\mathrm{T}_{\mathrm{c}}$. Further consequences of this multicomponent approach as, e.g., isotope effects, are discussed.

\section{Introduction}

In this review the complexity of the physics of HTSC is addressed. Starting point is a summary of characteristic properties of conventional superconductors which are compared to the more exotic behaviour observed in cuprates. Various crucial experimental results for cuprates are presented after that comparison, followed by an analysis of cuprates in terms of a multicomponent model. Here first a model of coexisting itinerant electrons and local pairs is introduced, followed by a more general two-component scenario. Results and conclusions are given in the end.

\section{Comparing conventional (BCS-type) superconductors with HTSC}

Conventional BCS type superconductors [1] exhibit three characteristic length scales, i.e. the London penetration depth $\lambda_{\mathrm{L}}$, the pair coherence length $\xi$, and the electron mean free path $l$. While the penetration depth indicates how deep a magnetic field may penetrate into a superconductor, the coherence length is a measure of the extensions of the paired state. Both quantities are related to each other through the electron mean free path $l$. The orders of magnitudes of all three quantities can be derived from the BCS Hamiltonian which is a consequence of the assumption that the electron pairing is mediated through the lattice. The lattice interaction $V_{\text {electron-phonon }}$ thus acts like a glue and has to overcome the Coulomb repulsion $U_{\text {Coulomb }}$ to yield an effective attractive potential $V_{\text {electron-phonon }}+U_{\text {Coulomb }}=V_{\text {effective }}$. The simplest possible Hamiltonian to describe the electron pairing is represented by:

$$
H_{B C S}=\sum_{k} E_{k} c_{k}^{+} c_{k}+\sum_{k, k^{\prime}} V_{\text {effective }} c_{k^{\prime} \uparrow}^{+} c_{-k^{\prime} \downarrow}^{+} c_{-k \downarrow} c_{k \uparrow}
$$

where $c_{k}^{+}, c_{k}$ are momentum $k$ dependent electron creation and annihilation with band energy $E_{k}$. The important consequences of equ. 1 are that the system's energy is lowered as compared to the normal state by an energy gap $\Delta$ like:

$$
E_{0}=2 \sum_{k<k_{f}} E_{k}-\frac{1}{2} \Delta_{0}^{2} \sum_{k}\left[\Delta_{0}^{2}+E_{k}^{2}\right]^{-1 / 2}
$$

The formation of a gap in the energy spectrum has a variety of important consequences which can easily be verified experimentally. First, BCS theory predicts that the ratio of $\Delta$ over the transition temperature $\mathrm{T}_{\mathrm{c}}$ is a constant, i.e. $\frac{2 \Delta_{0}}{k_{B} T_{c}} \cong 3.52$. Then, the magnitude of $\Delta$ is given by the phonon energy, the effective attractive interaction and the electronic density of states $N_{0 \text { : }}$ 
$\Delta_{0} \cong \hbar \omega e^{-\frac{1}{N_{0} V}}$. The correlation length is determined through the energy gap like $\xi_{0}=\frac{2}{\pi} \frac{\hbar v_{F}}{\Delta_{0}}$, where $v_{F}$ is the Fermi velocity. The energy gap can be measured by various methods and to mention a few only: specific heat, the tunnel effect, magnetic relaxation, ultrasound, and microwave absorption are extremely sensitive to it. The key ingredient in BCS theory is the prediction that the pairing glue stems from the lattice and this has been shown to be indeed true, since it implies that the transition temperature depends on the ionic mass like: $k_{B} T_{c} \approx M_{\text {ion }}{ }^{-\alpha}, \quad \alpha=0.5$. Most of the conventional superconductors show this isotope effect and the deviations from it can be explained within strong coupling theories. The success of the BCS theory is rather amazing since a variety of simplifications are introduced which nearly never apply to realistic systems, as, e.g., one-phonon exchange, spherical Fermi surface, simple lattice structure. These simplifying assumptions have, however, intrigued the conclusion, that superconductivity should best be found in at most binary systems with simple structures and well developed metallic properties.

HTSC compounds are - on the opposite - extremely complex materials with complicated structures, nearly 2D bad metallic properties with strongly anisotropic conductivities, short coherence lengths. The gap to $T_{c}$ ratio as well as the isotope effect on $T_{c}$ and the coherence factors deviate strongly from BCS predictions. In addition, it has been shown that conventional phonon mediated pairing can not be strong enough to account for the observed high values of $T_{c}$. These observations have led to the conclusion that the pairing mechanism should be different from the BCS one and that phonons may be silent here. A variety of exotic pairing mechanisms have been proposed consecutively, a conclusion about the glue has not been achieved yet.

\section{The two-component approach}

Very early after the introduction of the BCS theory extensions of it have been proposed to account for the generally more complex band structure of superconductors as considered by BCS. Here a two-band model was the first approach to take novel features arising from multiple bands into account [2-4]. Applications to HTSC were introduced very early after their discovery and novel approaches as e.g. induced superconductivity have been discussed [5-7]. The Hamiltonian for a two-band superconductor reads [2-4]:

$$
\begin{aligned}
& H=H_{1}+H_{2}+H_{3} \\
& H_{1}=\sum_{k, \sigma}\left[\left(E_{1}(\vec{k})-\mu_{1}\right) a_{k, \sigma}^{+} a_{k, \sigma}+\left(E_{2}(\vec{k})-\mu_{2}\right) b_{k, \sigma}^{+} b_{k, \sigma}\right] \\
& H_{2}=-\frac{1}{V} \sum_{k \neq g}\left[V_{1} a_{k \uparrow}^{+} a_{-k \downarrow}^{+} a_{-g \downarrow} a_{g \uparrow}+V_{2} b_{k \uparrow}^{+} b_{-k \downarrow}^{+} b_{-g \downarrow} b_{g \uparrow}\right] \\
& H_{3}=-\frac{1}{V} \sum_{k \neq g}\left[V_{12}\left(a_{k \uparrow}^{+} a_{-k \downarrow}^{+} b_{-g \downarrow} b_{g \uparrow}+b_{k \uparrow}^{+} b_{-k \downarrow}^{+} a_{-g \downarrow} a_{g \uparrow}\right)\right]
\end{aligned}
$$

where $\mu_{i}$ is the chemical potential in band $\mathrm{i}=1,2$ with creation and annihilation operators $a^{+}$, $b^{+}, a, b$ and band energies $E_{\mathrm{i}} . V_{\mathrm{i}}$ are effective attractive interactions in the corresponding bands and $V_{\mathrm{ij}}$ is the interband interaction between the two bands which can be attractive as well as repulsive. By applying a standard Bogoliubov approximation to the above equations solutions for the coupled gap equations are obtained corresponding to: 
$\Delta_{2}=\frac{V_{2}}{2 V} \sum_{k} \frac{\Delta_{2}}{\varepsilon_{2}(\vec{k})} \tanh \left[\frac{\varepsilon_{2}(\vec{k})}{2 k_{B} T_{c}}\right]+\frac{V_{12}}{2 V} \sum_{k} \frac{\Delta_{1}}{\varepsilon_{1}(\vec{k})} \tanh \left[\frac{\varepsilon_{1}(\vec{k})}{2 k_{B} T_{c}}\right]$

$k_{B} T_{c}=1.14 \hbar \omega \exp (-1 / \lambda)$

$1 / \lambda=\frac{1}{2}\left[\lambda_{1}+\lambda_{2} \pm \sqrt{\left(\lambda_{1}-\lambda_{2}\right)^{2}+4 \lambda_{12}^{2}}\right] /\left[\lambda_{1} \lambda_{2}-\lambda_{12}^{2}\right]$

where $\lambda_{i}=N_{i}(0) V_{i}$. Kresin and Wolf were probably the first who applied the two band model to HTSC. They calculated the gaps as functions of temperature and evaluated the tunnelling spectra. Extensions of the model to include multiple bands have been subsequently considered $[8,9]$ where special emphasis has been put on $T_{c}$ enhancement effects due to the interband coupling.

\section{The two-component model of coexisting local pairs and itinerant electrons}

In classical BCS-like superconductors and also two-band superconductors with finite interband interactions, the electron (hole) pairing temperature $\mathrm{T}_{\mathcal{C}}$ coincides with the formation of a coherent macroscopic quantum state. However, the pair formation could be independent of temperature of the long-range coherence and occur at much higher temperatures than the superconducting $T_{\mathcal{C}}$ [10-12]. In the case of strong electron-phonon interaction the carriers can form small polarons. Typically the strong lattice deformation is sufficient to attract another of these objects to combine into pairs which have Bose character and can eventually condense into a superfluid state [10]. The result is the formation of a macroscopic coherent quantum state of $\mathrm{q}=0$ eigenstates of hard-core bosons on a lattice. Materials exhibiting such a behavior should have paired electrons already in the normal state. Besides of strong electron-lattice interaction effects also an attractive Hubbard term could give rise to the above scenario [12], where the source of this attractive interaction has to be detailed and can - in principle - arise from all kinds of couplings. Such a system is described by the Hamiltonian [10-13]:

$$
\begin{aligned}
H= & \sum_{k, \sigma}\left(\varepsilon_{k}-\mu\right) c_{k, \sigma}^{+} c_{k, \sigma}+2 \sum_{i}\left(\Delta_{0}-\mu\right) b_{i}^{+} b_{i}-\sum_{i j} J_{i j} b_{i}^{+} b_{j}+ \\
& \sum_{k, q}\left[V_{q}(k) c_{k+q / 2, \uparrow}^{+} c_{-k+q / 2, \downarrow}^{+} b_{q}+\text { h.c. }\right]+H_{C}
\end{aligned}
$$

where $b^{+} b=n_{b}$ is the site $i$ dependent pair density with energy level $\Delta_{0}$ and hopping integral $J_{i j}$. The interaction between the local pairs and the itinerant $c$-electrons is given by $V$. Within such a scenario basically three different cases can be distinguished [14], i.e.:

i) $\quad \Delta_{0}<0$ : At $\mathrm{T}=0$ all available electrons form local pairs $\left(2 n_{b}>>n_{c}\right)$. This limit is the local pair regime (LP).

ii) $\quad \Delta_{0}>0$ : The $c$-electron band is filled up to the Fermi level $\mu=\Delta_{0}$ and the remaining electrons form local pairs. Obviously here a coexistence of local pairs and $c$ electrons is observed $\left(0<2 n_{b}, n_{c}<2\right)$ corresponding to a mixed regime $(\mathrm{LP}+\mathrm{E})$.

iii) $\quad \Delta_{0}>0$ : The Fermi level is below $\Delta_{0}$ and at $\mathrm{T}=0$ all available electrons are in $c$ states $\left(n_{c}>>2 n_{b}\right)$. This limit corresponds to $c$-regime or the weak-coupling (“BCS"-like) regime. 
The consequences for the superconducting properties of the above limiting cases are.

i) The local pair mobility is provided through virtual excitations into empty $c$ electron states. This mechanism gives rise to long range hopping of local pairs (which is analogue to the RKKY interaction for the s-d mechanism in the magnetic equivalent). The superconducting properties resemble those of a pure bipolaronic superconductor $[12,15,16]$.

ii) Superconductivity is a consequence of the interchange between local pairs and $c$ electron pairs. The $c$-electrons become "polarized" into Cooper pairs and local pairs gain mobility by decaying into $c$-electron pairs. In this intermediate regime neither the usual BCS picture nor the LP approach apply, and superconductivity has a "mixed" character. The system shows here features which are intermediate between both limits. This concerns the energy gap in the single electron excitation spectrum, the gap to $\mathrm{T}_{\mathrm{C}}$ ratios, the critical fields, the Ginzburg ratio $\kappa$, as well as the normal state properties.

iii) The situation is similar to that of a BCS superconductor: Cooper pairs of $c$ electrons are exchanged via virtual transitions into local pairs. This mechanism can be particularly effective in the presence of additional attractive interactions among the $c$-electrons.

The phase diagram of the mixed system depends crucially on the position of the local pair level and a changeover from a non-metallic state to a metallic one is observed with increasing $\Delta_{0}$ where an intermediate regime opens, which exhibits a pseudogap regime and superconductivity. This regime is characterized by the coexistence of preformed local pairs and paired $c$-electrons. The transition temperature $\mathrm{T}_{\mathrm{c}}$, the energy gaps and the coherence lengths also exhibit interesting crossovers from BCS to Bose-Einstein (BE) where especially the coherence length starts to decrease strongly when approaching the coexistence regime. Other consequences are summarized as follows [13, 14]:

i) In the mixed regime the mechanism of induced superconductivity (at constant $\Delta_{0}$ ) is not very sensitive to the pairing symmetry; $n_{\mathrm{c}}$ is nearly constant and $n_{\mathrm{b}}$ increases with $n$. The chemical potential in the superconducting phase remains almost pinned around $\Delta_{0}$.

ii) Well defined Bogoliubov quasiparticles can exist in the superconducting ground state. However, above $\mathrm{T}_{\mathrm{c}}$ (in the mixed regime) local pairs coexist with itinerant fermions, and the normal state properties deviate from Fermi liquid behavior.

iii) The $\mathrm{T}_{\mathrm{C}}$ 's as calculated beyond BCS-MFA within the T-matrix approach in $3 \mathrm{D}$ and in the Kosterlitz-Thouless (KT) scenario for 2D show crucial effects of pair (and phase) fluctuations in the mixed and LP regimes.

iv) In the mixed regime, for temperatures between $\mathrm{T}_{\mathrm{c}} \mathrm{MFA}$ and $\mathrm{T}_{\mathrm{C}} \mathrm{KT}$, the system exhibits a pseudogap in the $c$-electron spectrum, which develops into a real gap when moving to the LP regime. For $\Delta_{0}<0$, the LP's coexist with preformed $c$ electron pairs which have a binding energy $\mathrm{Eb}_{\mathrm{b}} / 2 \sim \mathrm{T}_{\mathrm{c}} \mathrm{MFA}$, and the system will

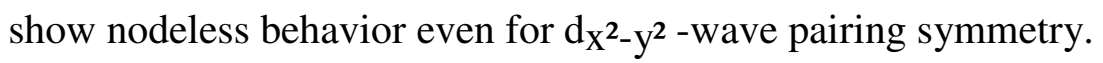

v) The existence of nodal quasiparticles for d-wave pairing (beyond the LP regime) gives rise to a linear in T relationship in the superfluid density which is absent for the other here considered pairing symmetries. The normalized superfluid stiffness as a function of normalized temperature shows only a weak dependence on the total carrier concentration.

vi) The gap to $\mathrm{T}_{\mathrm{c}}$ ratios differ substantially from weak coupling BCS predictions and are especially enlarged over the BCS value when d-wave symmetry is realized. 


\section{Experimental evidences for inhomogeneity, unconventional isotope effects and two component behavior}

The doping dependent phase diagram of HTSC is extremely complex since not only a crossover from an insulating antiferromagnet to a superconductor takes place, but in addition a pseudogap phase is observed which sets in at temperatures $T^{*}$ much higher than $\mathrm{T}_{\mathrm{c}}$ (Figure 5.1).

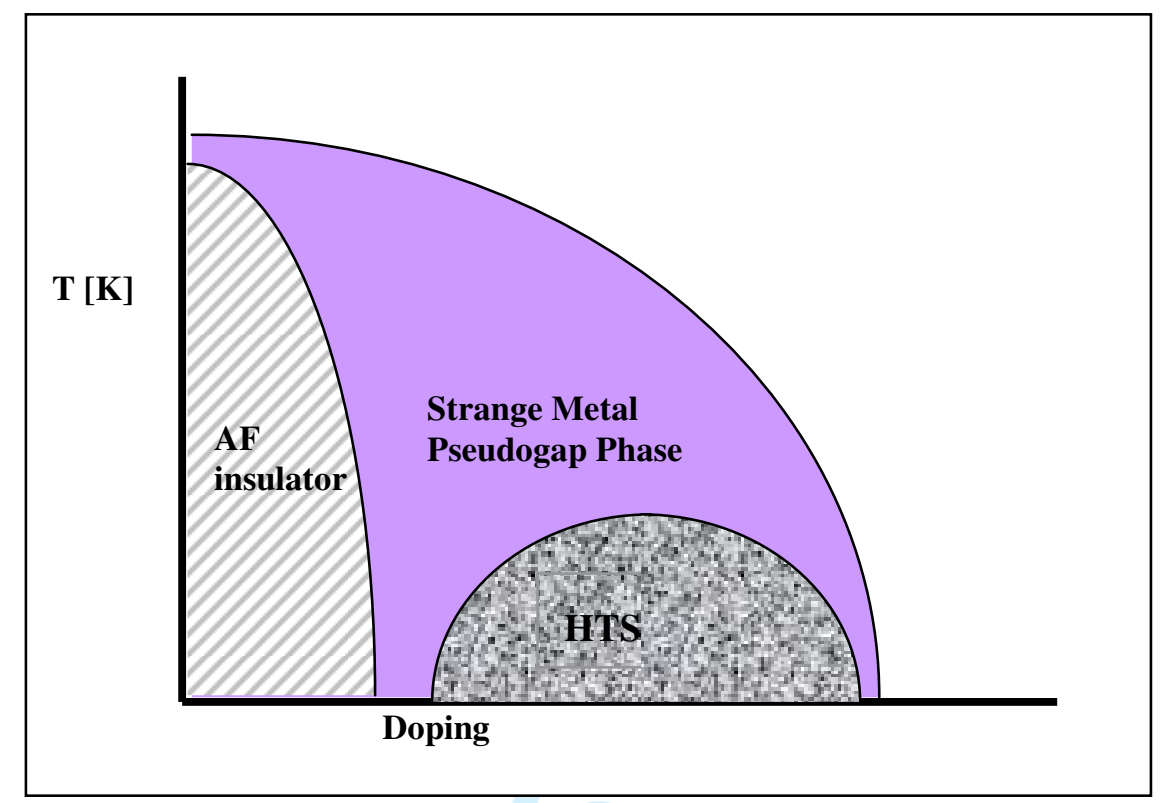

Figure 5.1 The doping dependent phase diagram of HTSC

Opposite to predictions from BCS theory, the isotope effect on $T_{c}$ is not a constant but is strongly doping dependent [17-19]: at optimum doping, i.e. maximum $\mathrm{T}_{c}=\mathrm{T}_{\mathrm{c}}{ }^{\mathrm{m}}$ within one family, it nearly vanishes, but increases to the BCS value - even sometimes exceeding it when approaching the antiferromagnetic regime. It should be noted here, that the vanishing isotope effect at $T_{c}{ }^{m}$ has frequently be taken as evidence that the pairing mechanism in HTSC is not phonon mediated but has to be attributed to strong correlations, which, indeed, govern the antiferromagnetism of the undoped parent compounds.

An even greater puzzle than the isotope effect on $T_{c}$ is the recently discovered one on $T^{*}$ [20-22]. Here the isotope effect is giant - one of the largest ever observed in solid state systems - and has most interestingly the reversed sign, i.e. $\mathrm{T}^{*}$ increases when replacing ${ }^{16} \mathrm{O}$ by ${ }^{18} \mathrm{O}$. A consistent explanation for this effect is missing, but it has been shown, that nonlinearity and strong anharmonicity could be its origin [23].

Nearly a decade ago EXAFS experiments have shown [24] that the local structure of HTSC deviates strongly from the average one, to be organized in a stripe like pattern of locally distorted areas coexisting with undistorted ones. The stripe formation has afterwards been confirmed by a variety of different methods. An explanation of this "phase separation" is still missing and it remains unclear whether it is in competition to superconductivity or supports it.

The displacement pattern involved in the local distortions has recently implicitly be identified from EPR measurements [25] to correspond to the quadrupolar distortion which is related to a Jahn-Teller type mode. The inhomogeneity of HTSC has also been directly observed by STM [26], where a local variation in the energy gap was reported [27]. Further evidence for multicomponent superconductivity stems from Andreev reflection measurements [28], which reveal that the superconducting order parameter consists of a leading d-wave and an additional s-wave component. 
All the above mentioned experiments shed some doubt on the interpretation of HTSC in terms of a purely electronically driven pairing mechanism [29]. Further support for a break down of t-J type models stems from ARPES results which a reveal unique kink in the electronic dispersion, attributed to strong electron phonon coupling [30]. An even more obvious result supporting a lattice mediated pairing mechanism than the kink structure, is the observation of an isotope effect on the London penetration depth $\lambda_{\mathrm{L}}$ [31]. Since this isotope effect is not present within conventional BCS theory, the role of the lattice to HTSC is different from the known phonon mediated scenario [32]. Support for an unconventional lattice mediated pairing mechanism comes also from strain effects on $\mathrm{T}_{\mathrm{c}}$. Substantial enhancements of it are obtained when strain is applied to films as well as to bulk material [33, 34]. There are - of course - a variety of other experimental results which have not been mentioned here, which give clear evidence that the pairing mechanism in HTSC is not due to strong correlations alone, but that the systems have to be treated as composites, where the doped holes surrounded by a lattice distortion are embedded in an antiferromagnetic matrix, which - in turn - is substantially coupled to the lattice through the strain fields of the local distortions: "spin-charge-lattice polarons".

\section{Multicomponent superconductivity: Interplay of spin, charge and lattice}

The superconducting regime of copper oxides emerges from the half-filled band antiferromagnetic insulating parent compounds with doping. Intuitively these should be metallic, are however Mott-Hubbard insulators due to the large Hubbard $\mathrm{U}$ at the $\mathrm{Cu}$ site. Estimates of $\mathrm{U}$ range between 5 and $8 \mathrm{eV}$ and thus set the largest energy scale in the physics of HTSC. This has consequently been taken as evidence that the superconducting regime is also governed by $\mathrm{U}[29]$ and that purely electronic models like the t-J model are sufficient to understand HTSC [35]. As has been shown above, there is a variety of experimental evidence that a crucial role is played by the lattice, even though its energy scale is of the order of $100 \mathrm{meV}$, only. However, opposite to conventional superconductors, the cuprates are extremely bad metals with conductivities an order of magnitude smaller than ordinary metals and giant anisotropy in it. This observation suggests that in the superconducting regime localized and itinerant states coexist. Yet the question remains, what are the localized and what are the itinerant states. The best starting point to understand the physics are the undoped parent compounds which can be described by the following Hamiltonian:

$$
H=\sum_{i, \sigma} \varepsilon_{d} d_{i, \sigma}^{+} d_{i, \sigma}+\sum_{i, \sigma} \varepsilon_{p} p_{i, \sigma}^{+} p_{i, \sigma}+U \sum_{i} d_{i \uparrow}^{+} d_{i \uparrow} d_{i \downarrow}^{+} d_{i \downarrow}+\sum_{i, \sigma} \sum_{l \in\{i\}} V_{i i} d_{i, \sigma}^{+} p_{l, \sigma}+H . c .
$$

where $d_{i, \sigma}^{+}, p_{i, \sigma}^{+}$creates a hole at site $i$ with spin $\sigma$ in the copper $d_{x^{2}-y^{2}}$, oxygen $p_{x}, p_{y}$ states, respectively,. $U$ is the on-site Coulomb repulsion at the copper site and $V$ measures the overlap between the copper and oxygen ion wave functions [35]. The model equ. 6.1 can be mapped on a single band model by projecting out triplet states and ignoring other than nearest neighbor hopping integrals [35]. Even though essential physics are lost by this procedure, the model has frequently been used to describe HTSC.

Upon doping, the holes randomly enter the antiferromagnetic matrix and induce a pronounced charge and lattice mismatch there which can be compensated by forming local distortions around the doped holes. Since these are accompanied by huge strain fields, also the spins are affected by it. Correspondingly, in the doped case the above Hamiltonian has to be complemented by terms which reflect these facts, i.e. electron-spin-lattice coupling has to be included in the spin and charge channel [32]:

$$
H_{c h-L}=\sum_{i, j, \vec{r}=\vec{x}, \vec{y}}\left[\gamma u_{i j, r} p_{i, \sigma}^{+} p_{j, \sigma}+\text { h.c. }\right]
$$




$$
\begin{aligned}
& H_{s p-L}=\sum_{i, j, \vec{r}=\vec{x}, \vec{y}}\left[u_{i j, r} d_{i, \sigma}^{+} d_{j, \sigma}+\text { h.c. }\right] \\
& H_{L}=\sum_{i, \vec{r}=\vec{x}, \vec{y}}\left[\frac{p_{i, r}^{2}}{2 M}+\frac{M}{2} \omega^{2} u_{i, r}^{2}\right]
\end{aligned}
$$

$H_{c h-L}$ and $H_{s p-L}$ are a consequence of charge-, spin- lattice coupling proportional to $\gamma$, where $u_{i, j}$ are the oxygen ion displacements along $\vec{r}=\vec{x}, \vec{y}$, the unit vectors along the axis directions, and $p, \omega$ are the momentum and frequency of the oscillator $i$ with mass $M$. The electronphonon coupling in equ. 6.2 is standard and can have multiple consequences. However, only few scenarios exist which have profound consequences for the ground state properties of the considered system:

i) Electron-phonon coupling may cause the formation of a charge density wave instability [36].

ii) A superconducting state may result in the sense of the BCS mechanism.

iii) A Peierls instability can occur [37].

iv) A combined charge-spin-density-wave instability could set in [38].

v) Polaron formation may take place [39].

One can systematically rule out various consequences from the above choices:

i) A structural instability is expected.

ii) Superconductivity is accompanied by a doping independent isotope effect, and an isotope effect on the penetration depth does not exist.

iii) A Peierls instability is, like i), accompanied by a structural phase transition.

iv) A combined charge-spin density wave instability competes with superconductivity.

v) Polaron formation is neither in contrast to superconductivity nor does it lead to structural transformations as long as it remains dynamic and local.

Consequently, in the following the focus will be on polaronic effects within the extended Hamiltonian equ. 6.1, which are manifold [40, 41], but, for brevity, only the most important ones within the context of isotope effects are discussed which occur in the hopping integrals. Both band energies, the doped hole band and the spin related one, have the same momentum $\mathrm{k}$ dependent dispersion, however with different k-space weight, since both are assumed to be mostly relevant to the $\mathrm{CuO}_{2}$ planes, namely:

$E_{k}=-2 t_{1}\left(\cos k_{x} a+\cos k_{y} b\right)+4 t_{2} \cos k_{x} a \cos k_{y} b-\mu$,

with $a \neq b$ being the in-plane lattice constants and $t_{1}, t_{2}$ are nearest and next nearest neighbour hopping integrals, respectively, and $\mu$ is the chemical potential which controls the number of particles, and has been shown to scale linearly with doping [42]. Phonon degrees of freedom appearing in Hamiltonian equ.6.2, can be eliminated by standard techniques [34]. An important term to mention, which appears in the transformed system is a spin charge coupling proportional to $V_{s p-c h} n_{c h} n_{s p}$ [40] together with a renormalization of the band energies:

$$
\begin{aligned}
\tilde{E}_{s p, c h}= & -2 t_{1} \exp \left[-\gamma^{2} \operatorname{coth} \frac{\hbar \omega}{2 k T}\right]\left(\cos k_{x} a+\cos k_{y} b\right)+ \\
& 4 t_{2} \exp \left[-\gamma^{2} \operatorname{coth} \frac{\hbar \omega}{2 k T}\right] \cos k_{x} a \cos k_{y} b-\Delta *-\mu,
\end{aligned}
$$

where $\Delta^{*}$ is the energy shift induced by the displacement $u_{i}$ of the nearest neighbour oxygen ions surrounding the copper ion. The focus here, however, is the polaronic band narrowing proportional to $\gamma$.

The model to be discussed is a coupled spin-charge phonon system with strong phonon mediated interband interactions between the spin and the charge channel [40]. Superconductivity may arise here from either attractive interactions in the spin channel, mostly mediated by antiferromagnetic fluctuations, or from electron-lattice interactions within 
the charge channel, or, finally, from a combined cooperative effect between both subsystems. Details of how to map the resulting Hamiltonian onto an effective two-band BCS type Hamiltonian have been given previously [43] and are not repeated here. In the following it is assumed that within this two-band model attractive pairing interactions stem from the spin channel whereas those in the charge channel are too weak to induce superconductivity here. The interband interactions between both components are the important terms which induce superconductivity also in this channel. Superconducting properties of the coupled charge spin - lattice system are studied within an effective two-band Bogoliubov quasiparticle approach. The resulting scenario is a two gap superconducting state, analogous to $\mathrm{MgB}_{2}$ [44], with the distinction that the order parameters are of different symmetries. In addition, and opposite to $\mathrm{MgB}_{2}$, in cuprates mostly a time-averaged gap is observed caused by fast fluctuations [45]. The superconducting gaps $E_{g, s}$ (s-wave gap), $E_{g, d}$ (d-wave gap) have been calculated as functions of doping for various coupling constants $\gamma$ and fixed ratio of $t_{2} / t_{1}=-0.3$ as suggested by band structure calculations for YBCO [46]. Figure 6.1 shows the individual gaps as a function of doping for various coupling constants. The ratio of the individual gaps with respect to the average one, namely $E_{g}=\sqrt{E_{g, s}^{2}+E_{g, d}^{2}}$, is nearly doping and coupling independent and exhibits values which are very close to those of ref. 28.

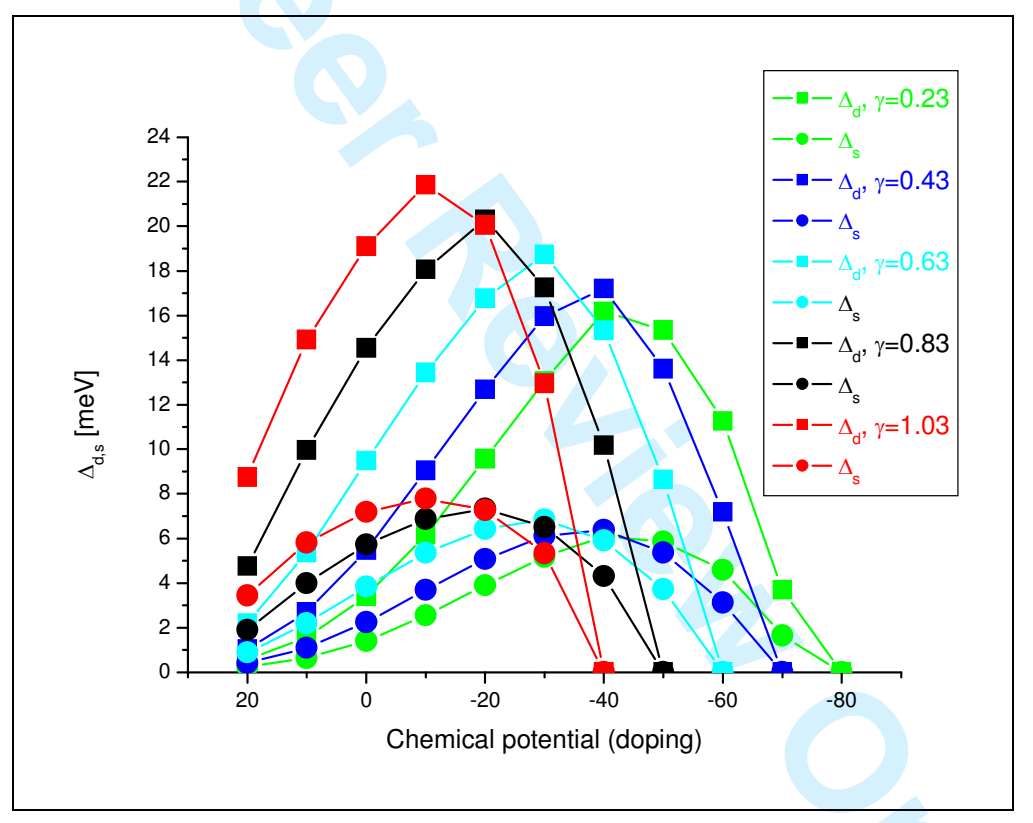

Figure 6.1 The calculated energy gaps as functions of doping for various polaronic coupling constants as indicated in the figure

Figure 6.2 shows the average gap $E_{g}=\sqrt{E_{g, s}^{2}+E_{g, d}^{2}}$ as a function of the corresponding $\mathrm{T}_{\mathrm{c}}$ where experimental data points for $\mathrm{Y}_{1-\mathrm{x}} \mathrm{Ca}_{\mathrm{x}} \mathrm{Ba}_{2} \mathrm{Cu}_{3} \mathrm{O}_{7-\delta}$ [28] have been added for direct comparison. 


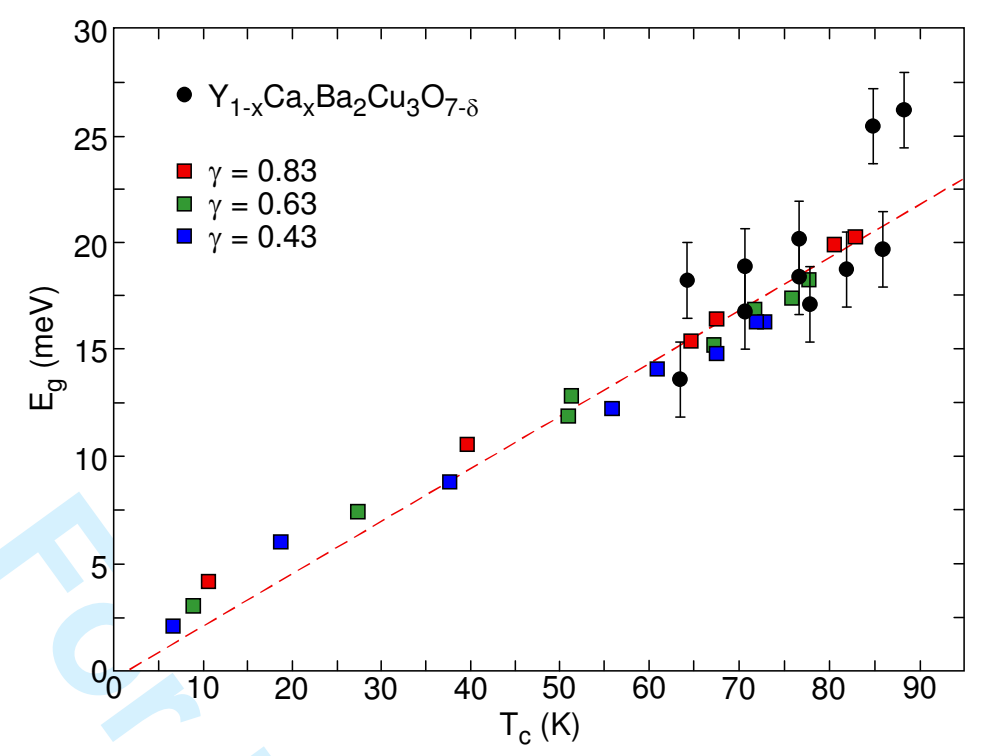

Figure 6.2 The calculated average energy gap (squares) as a function of the corresponding $T_{c}$ for various coupling constants as indicated in the figure. For comparison experimental data (black symbols) from Ref. 28 have been added. The straight dashed line is a guide to the eye.

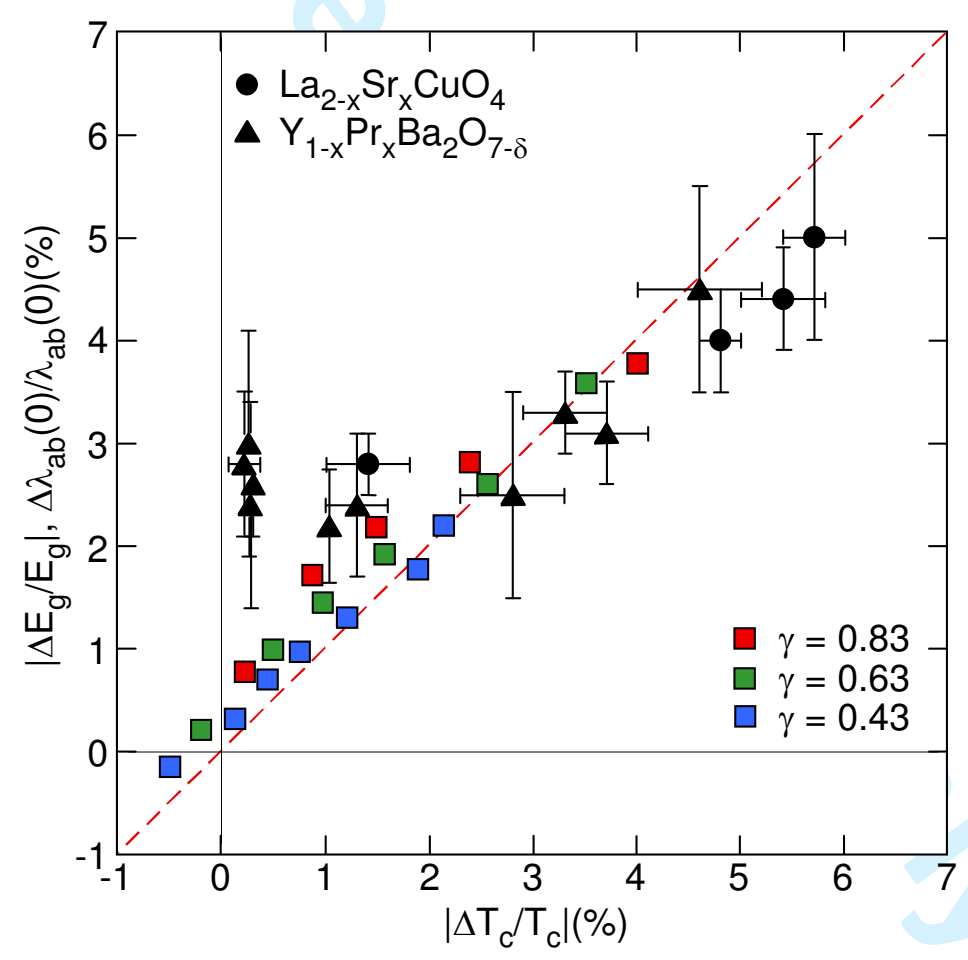

Figure 6.3 Relative isotope shift of the average gap $E_{g}=\sqrt{E_{g, d}^{2}+E_{g, s}^{2}}\left(\left|\Delta \mathrm{E}_{\mathrm{g}} / \mathrm{E}_{\mathrm{g}}\right|\right)$ as a function of the relative isotope shift of the superconducting transition temperature $\mathrm{T}_{\mathrm{c}}\left(\left|\Delta \mathrm{T}_{\mathrm{c}} / \mathrm{T}_{\mathrm{c}}\right|\right)$. Both gaps, the s-wave gap $E_{g, s}$ and the d-wave gap $E_{g, d}$ show nearly the same isotope shift. Squares are calculated values with $\gamma=0.43,0.63,083$, respectively. The dashed line is a guide to the eye. Full triangles and circles refer to experimental oxygen-isotope effect data of the zero-temperature in-plane magnetic penetration depth $\lambda_{a b}(0)$ and $T_{c}$ taken from Ref. 31 . The ratio of $\mathrm{t}_{2} / \mathrm{t}_{1}=-0.3$ in all calculations and the corresponding figures. 
Furthermore, the oxygen-isotope $\left({ }^{16} \mathrm{O} /{ }^{18} \mathrm{O}\right)$ effects on $E_{g}$ and $\mathrm{T}_{\mathrm{c}}$ have been calculated for various values of $\gamma$ and the same parameters as in Fig 6.1. As shown in Fig. 6.3, the relative isotope shifts $\left|\Delta \mathrm{E}_{\mathrm{g}} / \mathrm{E}_{\mathrm{g}}\right|=\left|\left({ }^{18} \mathrm{E}_{\mathrm{g}}-{ }^{16} \mathrm{E}_{\mathrm{g}}\right)\right|{ }^{16} \mathrm{E}_{\mathrm{g}} \mid$ and $\left|\Delta \mathrm{T}_{\mathrm{c}} / \mathrm{T}_{\mathrm{c}}\right|=\left|\left({ }^{18} \mathrm{~T}_{\mathrm{c}}-{ }^{16} \mathrm{~T}_{\mathrm{c}}\right) /{ }^{16} \mathrm{~T}_{\mathrm{c}}\right|$ are found to be equal (dashed line). This striking finding is in excellent agreement with experimental data [31] of the oxygen-isotope effect on the zero-temperature in-plane magnetic penetration depth $\lambda_{\mathrm{ab}}(0)$ and $\mathrm{T}_{\mathrm{c}}$ also included in Fig. 6.3 for direct comparison. However, the saturation effects observed in $\Delta \lambda_{\mathrm{ab}}(0) / \lambda_{\mathrm{ab}}(0)$ at optimum doping are not found for $\left|\Delta \mathrm{E}_{\mathrm{g}} / \mathrm{E}_{\mathrm{g}}\right|$ and appear only if $t_{1} / t_{2}$ is strongly enhanced as compared to the present value. The close resemblance between $\left|\Delta \mathrm{E}_{\mathrm{g}} / \mathrm{E}_{\mathrm{g}}\right|$ and $\Delta \lambda_{\mathrm{ab}}(0) / \lambda_{\mathrm{ab}}(0)$ in Fig. 6.3 is not accidental since in both quantities the leading term for the isotope effect stems from the band energies.

Within the suggested scenario an isotope effect on $\mathrm{T}_{\mathrm{c}}$ can only be caused by the band narrowing effect proportional to $\gamma$. This effect is present in both hopping integrals and treated on equal footings. It may, however, arise from the individual contributions stemming from $t_{1}$, $t_{2}$ alone, and thus give a clue to the identification of the underlying symmetry of the lattice mode which is involved here. The total isotope effect from both, $t_{1}$ and $t_{2}$, and the individual contributions to it are shown in fig. 6.4 together with the experimental data.

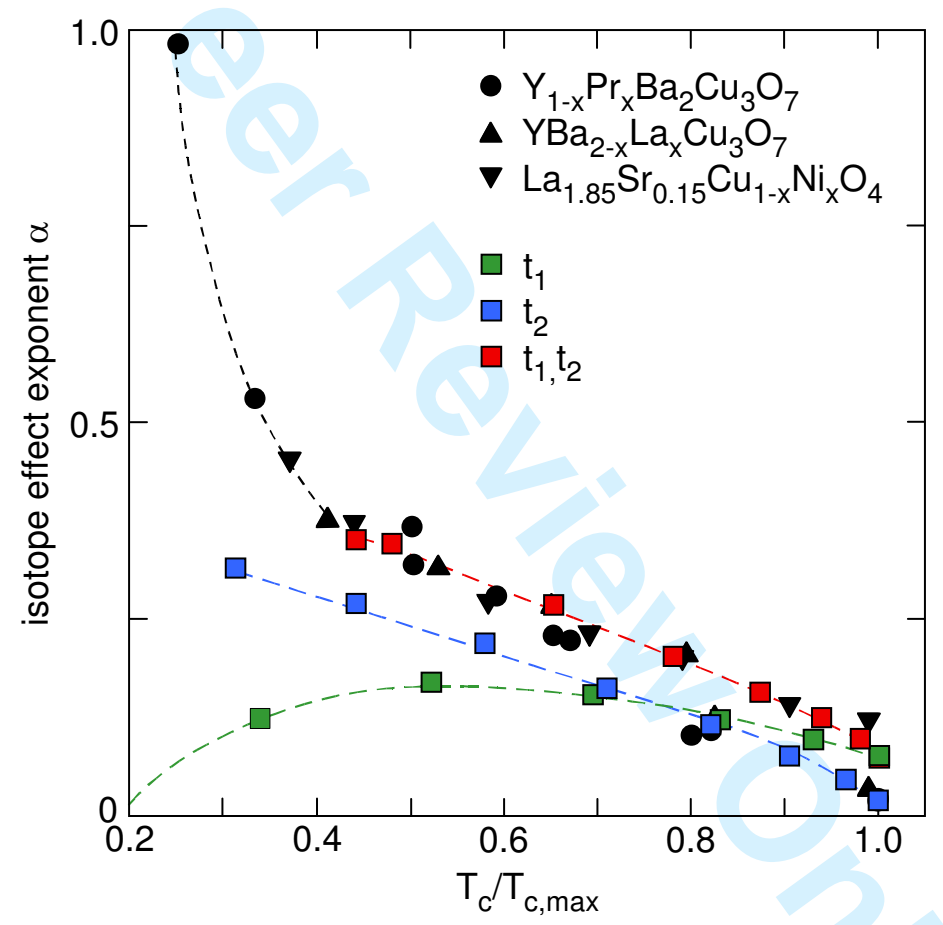

Figure 6.4 The oxygen isotope exponent $\alpha$ as a function of $\mathrm{T}_{\mathrm{c}}{ }^{\mathrm{m}} / \mathrm{T}_{\mathrm{c}}$. Squares refer to calculated values where the red squares are obtained by renormalizing both, $t_{1}$ and $t_{2}$, the blue ones by renormalizing $t_{2}$ only, and for the green ones only $t_{1}$ is renormalized. The black symbols are experimental data point taken from Refs. 17-19.

There, the total calculated isotope effect is in very good agreement with the experimental data. Opposite to this observation, the individual contributions behave quite differently, since the isotope effect stemming from $t_{1}$ only, first follows closely the experimental one but then starts to deviate strongly in the underdoped regime to even adopt negative values there. The effect from $t_{2}$ is different and shows the correct trend with doping. Since $t_{1}$ corresponds to the hopping along the $\langle 10\rangle,\langle 01\rangle$ direction and $t_{2}$ to the hopping along the diagonal $\left.<11\right\rangle$ direction, the above results admit to draw conclusion about the symmetry of the underlying lattice mode. Since the half- and full breathing modes as well as the buckling mode involve 
the nearest neighbour hopping term, these can be excluded to be the origin of the isotope effect on $T_{c}$ and $\lambda_{L}$. Dominated by second nearest neighbour hopping is the quadrupolar $\mathrm{Q}_{2}-$ type mode which is responsible for the Jahn-Teller (JT) effect in other copper compounds.

The model has also been shown to explain the isotope effect on the penetration depth and an interesting linear correlation between the isotope effect on $T_{c}$ and $\lambda_{L}$ is observed [47, 48], which shows that the isotope effects of both quantities have the same origin, namely the coupling of the electronic degrees of freedom to a quadrupolar Jahn-Teller type mode.

\section{Conclusions}

In this contribution it has been shown that natural extensions of the Hubbard model which incorporate the coupling to the lattice, are an essential ingredient for any model for high $\mathrm{T}_{\mathrm{c}}$ cuprates in order to understand the observed unconventional isotope effects. The most important findings are that the isotope effects are a consequence of polaron formation where the second nearest neighbour transfer integral $t_{2}$ causes the isotope effects. This observation suggests that the important lattice mode in this respect is a pseudo JT $\mathrm{Q}_{2}$-type mode as also suggested by chemical consideration [49] and rules out that half-, full-breathing and buckling modes are the origin of unconventional isotope effects within this two-dimensional approach.

Acknowledgement It is a pleasure to acknowledge many useful discussions with A. R. Bishop, H. Keller, R. Micnas, K. A. Müller and A. Simon.

\section{References}

1.) Bardeen J, Cooper NL, Schrieffer JR (1957) Phys Rev B: 108, 1175

2.) Suhl H, Matthias B, Walker L, (1959) Phys Rev Lett 3: 552

3.) Moskalenko V, (1959) Fiz Metal Metallov 8: 503

4.) Geilikman B, Zaitsev R, Kresin V, (1967) Fiz Tverd. Tela (Leningrad) 9: 693 [(1966) Sov Phys Solid State 9: 542]

5.) Kresin V, Wolf S, (1990) Physica C 169: 476; Kresin V, Wolf S, Deutscher G, (1992) J Phys C 191: 9

6.) Kresin V, Wolf S, (1990) Phys Rev B 41: 4278; Kresin V, (1987) Sol St Comm 63: 725; Kresin V, Wolf S, (1987) ibid. 63: 1141

7.) Kresin V, Wolf S, Novel Superconductivity, (1987) ed. Wolf S, Kresin V, Plenum, New York; Kresin V, Wolf S, (1992) Phys Rev B 46: 6458; Adrian SD, Wolf SA, Dolgov O, Shulga S, Kresin VZ, (1997) Phys Rev B 56: 7878;

8.) Bussmann-Holder A, Genzel L, Simon A, Bishop AR (1993) Z Phys B: 92,149

9.) Bussmann-Holder A, Micnas R, Bishop AR (2004) Europ Phys J 37: 345

10.) Robaszkiewicz S, Micnas R, Ranninger J, (1987) Phys Rev B 36: 180

11.) Ranninger J, Micnas R, Robaszkiewicz S (1988) Ann Phys (Paris) 13: 455

12.) Micnas R, Ranninger J, Robaszkiewicz S, (1990) Rev Mod Phys 62: 113 and refs therein

13.) Micnas R, Robaszkiewicz S, Bussmann-Holder A (2002) Phys Rev B 66: 104516 
14.) Micnas R, Robaszkiewicz S, Bussmann-Holder A (2005) Springer Series Structure and Bonding, Superconductivity in complex systems

15.) Micnas R, Robaszkiewicz S In High Temperature Superconductivity: 10 Years after the Discovery, Nato ASI series E, vol 343, eds Kaldis E, Liarokapis E, Müller KA (1997) Kluwer Academic, p31 and refs therein

16.) Micnas R, Robaszkiewicz S, (1992) Phys Rev B 45: 9900; Micnas R, Robaszkiewicz S, Kostyrko T, (1995) Phys Rev B 52: 6863

17.) Franck JP, in Physical Properties of high temperature superconductors IV (ed. Ginsberg, D. M.) (World Scientific, Singapore, 1994) 189-293

18.) Keller H (2005) Springer Series Structure \& Bonding, Superconductivity in Complex Systems

19.) Genzel L, Wittlin A, Bauer M, Cardona m, Schönherr E, Simon A (1989) Phys Rev B 40: 2170

20.) Lanzara A, Zhao G, Saini NL, Bianconi A, Conder K, Keller H and Müller KA, (1999) J Phys: Cond Mat 11: L545

21.) RubioTemprano D, Mesot J, Janssen S, Conder K, Furrer A, Sokolov A, Trounov V, Kazakov SM, Karpinski J and Müller KA, (2001) Eur Phys J B 19: R5

22.) RubioTemprano D, Mesot J, Janssen S, Conder K, Furrer A, Mutka H and Müller KA, (2000) Phys Rev Lett 84: 1999

23.) Bussmann-Holder A, Büttner H, Simon A, Bishop AR (2000) Phil Mag B 80: 1955

24.) Bianconi A, Saini NL, Rossetti T, Lanzara A, Perali A, Missori M, Oyanagi H, Yamaguchi Y, Nishihara Y, Ha DH, (1996) Phys Rev B 54: 12018

25.) Kochelaev BI, Sichelschmidt J, Elschner B, Lemor W, Loidl A (1997) Phys Rev Lett 79: 4274-4277

26.) Lang KM, Hoffman JE, Hudson EW, Uchida S, Davis JC (2002) Nature 415: 412

27.) Pan SH, O'Neal JP, Badzey RL, Chamon C, Ding H, Engelbrecht JR, Wang Z, Eisaki H, Uchida S, Gupta AK, Ng K-W, Hudson EW, Lang KM, Davis JC (2001) Nature 413: 282

28.) Kohen A, Leibowitch G, Deutscher G (2003) Phys Rev Lett 90: 207005

29.) Anderson PW (1987) Science 235: 1196

30.) Lanzara A et al (2001) Nature 412: 510

31.) Khasanov $R$, Eshchenko DG, Luetkens $H$, Morenzoni $E$, Prokscha T, Suter A, Garigianov N, Maili M, Roos J, Conder K,

Keller H, cond-mat/0305477 and (2004) Phys Rev Lett 92: 057602

32.) Bussmann-Holder A, Keller H, cond-mat/0409738

33.) Abrecht $\mathrm{M}$ et al., (2003) Phys Rev Lett 91: 057002

34.) Locquet J-P et al., (1998) Nature $394: 453$

35.) Zhang FC, Rice TM (1988) Phys Rev B 37: 3759

36.) Rice TM, Scott GK (1975) Phys Rev Lett 35: 120

37.) Pytte E (1974) Phys Rev B 10: 4637

38.) Bahara SN, Mishra SG (1985) Phys Rev B 31: 2773

39.) Lang SG, Firsov Yu A (1963) Sov. Phys. JETP 16,:1302 
40.) Bussmann-Holder A, Müller KA, Micnas R, Büttner H, Simon A, Bishop AR ((2001) J Phys: Cond Mat 13: L545

41.) Bussmann-Holder A, Bishop AR, Büttner H, Egami T, Micnas R, Müller KA (2001) J Phys: Cond Mat 13: L545

42.) Shen $\mathrm{KM}$ et al, cond-mat/0407002

43.) Bussmann-Holder A, Micnas R, Bishop AR (2004) Europ Phys J 37: 345

44.) Choi HJ, Roundy D, Sun H,. Cohen ML, Louie SG, (2002) Nature 418: 758

45.) Sudbo A, Chakravarty S, Strong S, Anderson PW (1994) Phys Rev B 49: 12245

46.) Pavarini E, Dasgupta I, Saha-Dasgupta T, Jepsen O, Andersen OK (2001) Phys Rev Lett 87: 047003

47.) Bussmann-Holder A, Keller H, Micnas R, Bishop AR, Müller $\mathrm{KA}$, to be published

48.) Bussmann-Holder A, Keller H, Müller KA (2005) Springer Series Structure and Bonding: Superconductivity in Complex Systems

49.) Simon A (1987).Angew Chem 26: 579 